\title{
Investigating the Effects of Natural and Artificial Sugars on the Rate of Growth and Respiration in Saccharomyces cerevisiae
}

\author{
Krishna Chaitanya Naredla ${ }^{1 *}$, Seema Gupta ${ }^{1}$ and Maisnam Jaya Devi ${ }^{2}$ \\ ${ }^{1}$ Paramita Group of School, Karimnagar, Telangana, 505001, India \\ ${ }^{2}$ Pesticide management division, National institute of Plant health management, \\ Rajendranagar, Hyderabad, 500030, India \\ *Corresponding author
}

\section{A B S T R A C T}

\begin{tabular}{|l|}
\hline Ke y w o r d s \\
$\begin{array}{l}\text { Saccharomyces } \\
\text { cerevisiae, } \\
\text { Aspartame, } \\
\text { artificial sweetener, } \\
\text { Growth rate, } \\
\text { Respiration }\end{array}$ \\
\hline Article Info \\
\hline $\begin{array}{l}\text { Accepted: } \\
\text { 14 December } 2018 \\
\text { Available Online: } \\
\text { 10 January } 2019\end{array}$ \\
\hline
\end{tabular}

\section{Keywords}

Saccharomyces cerevisiae, artificial sweetener, Growth rate,

\section{Introduction}

Saccharomyces cerevisiae, commonly referred as baker's yeast is a species of yeast. It is considered to be 'model organism' by the scientists as it is unicellular and eukaryotic also and this microbe is globular shaped yellow-green colored and belonging to fungi kingdom. It basically feeds on sugars, hence the name saccharomyces. It feeds on those sugars and converts them to cellular energy thorough anaerobic fermentation. This
Baking yeast is one of the most widely used fungi used in the food industry. During processing the food products many preservatives along with sweeteners are used in order to increase the shelf life of the product. Presently from few years non-caloric artificial sweeteners have replaced natural sugars in majority of the food industries. It is believed to have less calorie intake as compared with natural sugars. There are several works done on these sweeteners supporting and denying positive health conditions in man. Similarly, it is important to study any possible ill effects of these sweeteners on good microbes like Saccharomyces cerevisiae. In the present study it is found that artificial sweetener Aspartame when compared with natural sugars has no significant effect on rate of growth and respiration of Saccharomyces cerevisiae. 
There are various factors like temperature, $\mathrm{pH}$ and concentration of the substrate affecting the rate of respiration in $S$. cereviceae (Tilak W. Nagodawithana et al., 1974). Henceforth, any alteration in these factors may fluctuate the growth of the yeast and thereby the product quality. It has been observed that, $\mathrm{pH}$ of the medium suitable for growth of these fungi is between 2.75 to 4.25 and the optimum temperature is $27^{\circ} \mathrm{C}-30^{\circ} \mathrm{C}$ (R. C. Jones and J. S. Hough, 1970). S. cereviceae naturally grows on one of the most important food ingredients Saccharose known as table sugar or sucrose (Graeme M Walker and graham G Stewart, 2016). A disaccharide made of monosaccharide glucose and fructose. Sucrose is often extracted from sugar cane or sugar beet for human consumption. Sucrose is naturally formed by plants and cyanobacteria but not by other organisms (Rebecca J. Brown et al., 2010).

There are substitutes for these natural sugars to maintain the sugar level in the body and prevent various symptoms and diseases related to it. The molecules that are derived from sugars, usually used as sugar substitutes but consist of low calorie load. Commonly seen artificial sweeteners are Aspartame, Sucralose and Saccharin (Vikas Purohit and Sundeep Mishra, 2018). They are usually 200-600 times sweeter than normal sucrose. Aspartame is a dipeptide produced by equal combination of aspartic acid and methyl ester of L-phenylalanine by protease thermolysis. It is approved for human consumption and is widely used in soft drinks and as a low calorie artificial sweetener. There are many controversies on consumption of aspartame causing certain health issues like weight gain and heart disease risks (Annie Ferland et al., 2007). The other factor which can affect the growth and fermentation process by yeast is mineral form of nitrates like Sodium nitrate, a chemical compound soluble in water (Agata Swiecilo, 2008). It is a readily available source of nitrate anion, found in natural environment with large accumulation in the environment, used as food additives in the production of cheese and also as a color fixative in the meat curing industries. The mineral form of this salt is also known as nitratine or soda niter and sometimes also referred as Chile saltpeter.

Excessive usage of it is very harmful to the mammals and the repercussions are very disparaging. Studies show that it can cause stomach, colon cancers also in severe cases including the risk of Alzheimer's and Parkinson diseases also in extreme cases (Alicja Mortensen et al., 2017; Suzanne M. de la Monte et al., 2015). It is also used in the waste water industry for favoring the respiration of the facultative microorganisms. Sometimes used by the marine aquarists in utilizing the carbon dosing techniques, to increase the nitrate levels in the water promoting the bacterial growth and in making fertilizers ( Cheng Wei Lui et al., 2014).

This research is done to understand the changes in the rate of growth and respiration on $S$. cereviceae, along respond when the concentrations of the different types of sugars and salts are added to the intracellular environments.

\section{Materials and Methods}

\section{Strain for yeast (S. cereviceae)}

S. cereviceae strain was fetched from the baking yeast available in the local market at Telangana. Growth media for mother culture is prepared in the laboratory and cell line is maintained throughout the duration of study. Laboratory conditions were maintained along with hygiene to avoid the contamination of the culture to get accurate results. Autoclave (Labline, LAC-05, 5-20 lts) and laminar air flow (Horizontal laminar air flow, Glowmax 
engineers, 300 LUX) are used to sterilize the equipment and culturing of $S$. cereviceae.

\section{Growth media for $S$. cereviceae}

\section{Mother culture}

Pure cell line of yeast is inoculated and maintained in solid and liquid yeast extract peptone glycerol media (YPG). Cell count and density of the yeast in stock culture is maintained to $4.2 \times 10^{8} \mathrm{cell} / \mathrm{ml}$.

\section{Test cultures}

Three varieties of sugar namely Aspartame, wheat malt and glucose are tested on yeast cells to observe any evident changes in viability and rate of respiration rate. The study is done for $24 \mathrm{hrs}$. Duration under controlled laboratory conditions and observations were made for 3 hours interval.

\section{Preparation of media}

Yeast suspension of pure line from baking yeast is used to create mother culture and test with various sweeteners. Portions of the 0.1 $\mathrm{ml}$ of the yeast cell suspensions are inoculated into solid medium in Petri dish. The dishes are inoculated at $28^{\circ} \mathrm{C}, 80 \% \mathrm{RH}$ and $6.5 \mathrm{pH}$ for $48 \mathrm{hrs}$ the ideal regeneration time of the yeast cells is $48 \mathrm{hrs}$. The colonies grown in the plates were observed for viability and few of those cells are incubated in liquid suspension. Temperature was maintained at $25-32^{\circ} \mathrm{C}$ (Quincy lab $10 \mathrm{GC}, 0.7$ cubic feet) and relative humidity at $80 \%$ (Honeywell Humidastat $\mathrm{H6000}$ ) and $\mathrm{pH}$ is maintained at 6.5 (Sigma Aldrich buffer tablets) for both mother culture and test cultures. Mother culture for the yeast were stored at $10^{\circ} \mathrm{C}$ in refrigeration and used as per requirement of the experiments. The colonies in the plates along with the turbidities in the suspensions are recorded (Table 1).

Sampling and analysis
Yeast is added in the proportion of $1 \mathrm{gm}$ to all the flasks and mouths of flasks are affixed with balloons to each flask. Flasks are shifted to $60^{\circ} \mathrm{C}$ water bath. Now the balloons are removed from the setup and its necks were twisted and pinned to prevent leakage of accumulated gas. Plastic tubs are filled and cylinders are filled with water and sealed with wrap. Balloons are placed on calibrated cylinders attached with pressure gauge in such a way that gas in the balloons will enter into cylinder. As the mouth of the balloons is slowly released the gas pumped into the cylinders and the pressure was recorded. This gave us the accurate value of the gas formed after fermentation process by yeast in three different.

\section{Results and Discussion} Rate of growth and respiration for $S$.
cereviceae

Artificial sugars are referred by many producers to reduced intake of calories and glucose in the body. This may perhaps reduce the risk for diabetic and obese individuals and help them to have normal metabolism. Accordance with this food production industries have made it into use for various products. Some of these also undergo fermentation procedures including the bakeries having large scale use of yeast $S$. cereviceae. It is thereby important to analyze the effect of these artificial sweeteners on the useful fungi like yeast as it is wildly used in processing the food. In the present work we have observed the effect of various sugar samples including natural and artificial sweeteners on baking yeast. The two major prospective including growth rate (CFU) and rate of respiration is recorded for 24 hours of fermentation in different samples of sweetener. Balloon method is adapted to calculate the amount of ethanol produced by $S$. cereviceae and calibrated by pressure 
gauge (psi). According to the data analysis among the four sets of experiment, the growth rate is observed to be more in Aspartame as $0.69 \times 10^{-6} \mathrm{CFU}$ along with rate of respiration as $11.37 \mathrm{psi}$. This is followed by Malt sugar resulting growth rate as $0.71 \times 10^{-6}$ and rate of respiration as $10.91 \mathrm{psi}$ and Glucose showing growth rate as $0.73 \times 10^{-6} \mathrm{CFU}$ along with rate of respiration as 10.23 psi. Finally the controlled set is with the least value of growth rate as $0.34 \times 10^{-6} \mathrm{CFU}$ and rate of respiration as 06.15 psi (Table 2 and Graph 1).

Table.1 Composition of the test compounds including artificial and natural sweeteners

\begin{tabular}{|l|l|l|l|l|}
\hline \multicolumn{1}{|c|}{ Table } & \multicolumn{1}{|c|}{ Glucose $(\times \mathbf{6})$} & \multicolumn{1}{|c|}{$\begin{array}{c}\text { ASPARTAME } \\
(\times \mathbf{6})\end{array}$} & \multicolumn{1}{|c|}{ Malt sugar $(\times \mathbf{6})$} & \multicolumn{1}{c|}{$\begin{array}{c}\text { Control set } \\
(\mathbf{6 X})\end{array}$} \\
\hline Test compound & $1 \mathrm{gm}$ & $1 \mathrm{gm}$ & $1 \mathrm{gm}$ & Nil \\
\hline Stock solution & $2.25 \mathrm{ml}$ & $2.25 \mathrm{ml}$ & $2.25 \mathrm{ml}$ & $2.25 \mathrm{ml}$ \\
\hline Water & $22.5 \mathrm{ml}$ & $22.5 \mathrm{ml}$ & $22.5 \mathrm{ml}$ & $22.5 \mathrm{ml}$ \\
\hline Total & $25 \mathrm{ml}$ & $25 \mathrm{ml}$ & $25 \mathrm{ml}$ & $24 \mathrm{ml}$ \\
\hline
\end{tabular}

Table.2 Rate of growth and respiration for S. cereviceae post 24 hours of fermentation

\begin{tabular}{|r|l|c|c|}
\hline Sr.no & Treatments & $\begin{array}{l}\text { Growth rate after 24 } \\
\text { hours/ CFU count }\end{array}$ & $\begin{array}{l}\text { Rate of respiration } \\
\text { after 24 hours/ psi }\end{array}$ \\
\hline 1. & Glucose & $0.73 \times 10^{-6}$ & 10.23 \\
\hline 2. & Malt sugar & $0.71 \times 10^{-6}$ & 10.91 \\
\hline 3. & Aspartame & $0.69 \times 10^{-6}$ & 11.37 \\
\hline 4. & Control set & $0.34 \times 10^{-6}$ & 06.15 \\
\hline
\end{tabular}

Graph.1 Rate of growth and respiration by $S$. cereviceae in various samples of sweeteners

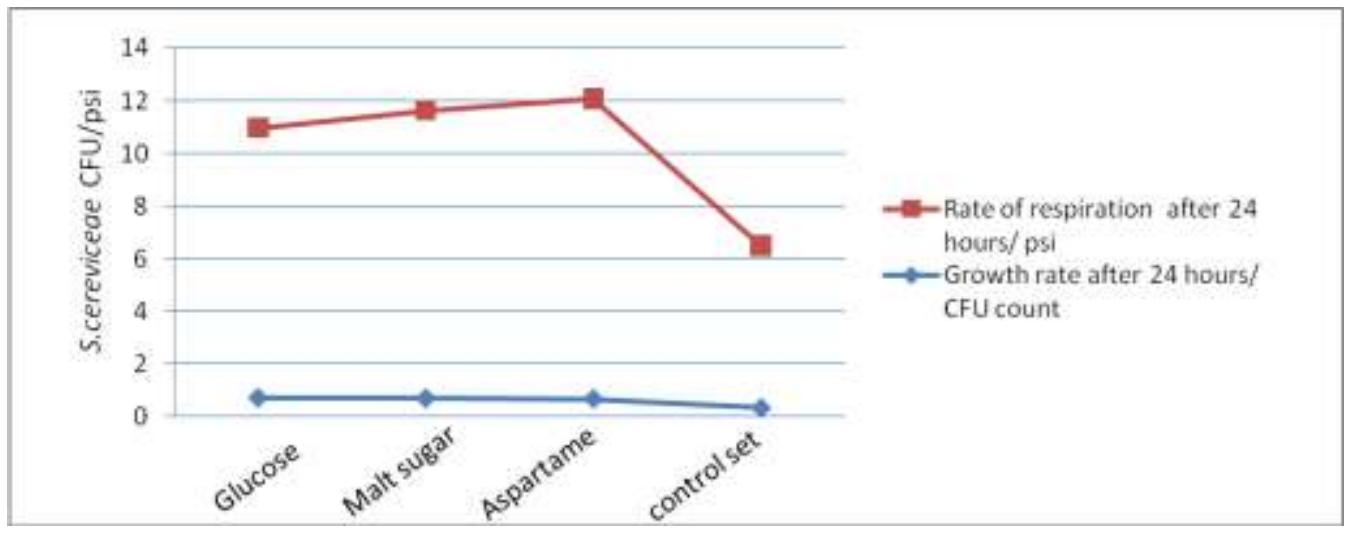

In recent years, the world is being more cautious about the health longevity through adapting various measures like naturopathy, yoga, balanced diet and so on. Among which the food industry is more linked up to these phenomenon to be followed, so as to attract more consumers for the products. In the similar verge it is observed that consumption of sugars is one of the main causes of various health hazards including diabetes, cardiac issues and even obesity. Thus, the researchers have come up with an alternative to this issue 
by introducing artificial sweeteners promising less calorie intake along with good health to the consumers. As Saccharomyces cerevisiae is one of the widely used microbe in the baking process, its activity in presence of various sweeteners is to be analyzed. In the present work the different samples of sweeteners such as aspartame, maltose and glucose are tested on rate of growth and respiration of Saccharomyces cerevisiae (Baking yeast). The observations made in 24 hours of the test period show negligible difference in values noted for artificial and natural sugars on activity of yeast cells. Similar observations were made by Abour et al., (2017) in their studies on effect of different sugars on respiration of yeast. Polakowski (2008) and Flushce et al., (2015) had similar studies on the effect of sugar from rice on respiration activity of yeast which was found to have nearly same values as that of artificial sugars. The researchers have studied similar comparisons on humans so as to conform activity of artificial and natural sugars in higher organisms. Gliemmo (2007) did a study on interactions of natural and artificial sugar in a living system (higher animals), according to his study both natural and artificial sugars interact with each other and have approximately similar activity on living cells. Marissa Fennell et al., (2016) observed that ethanol was formed more by artificial sugars in yeast culture than the natural sugar with a slight difference in the value of cellular respiration between the sugar samples. Sanchari Chattopadhyay et al., (2014) under the investigation studies on various sugars on activity of yeast observed least difference in activity of various sugars on rate of respiration of yeast cells. By this it is evident that there is not much difference made by natural and artificial sugars on growth and respiration rate of baking yeast, Saccharomyces cerevisiae.

In conclusion addition of the artificial sweetener namely aspartame and its comparative analysis with natural sugars like glucose and maltose in the growth medium is showing approximately similar results for the rate of growth and respiration in $S$. cereviceae. This could be considered as negligible effect of artificial sweeteners on these activities of useful fungi like Saccharomyces cerevisiae.

\section{Acknowledgement}

We thank the management of Paramita group of schools and specially Mrs. Rashmitha Prasad to take interest in the present work and encourage us for the same. We appreciate Krishna Naredla for his unconditional and insurmountable support for this work.

\section{References}

Agata swiecilo, 2008. Effect of sodium nitrate (V) on Saccharomyces cerevisiae strains of different Antioxidative status and energetic metabolism. Polish journal of food and nutrition science. Vol. 58, No. 1, pp. 41-44.

Annie Ferland, Patrice Brassard, Paul Poirier, 2007. Is Aspartame really safer in Reducing the risk of Hypoglycemia during exercise in patients with type 2 Diabetes? American Diabetes Associations. 30(7): e59.

Alicja Mortensen, Fernando Aguilar, Riccardo Crebelli and Alessandro Di Domenico, 2017. Re-evaluation of sodium nitrate (E 251) and potassium nitrate (E 252) as food and additives. EFSA journal. https://doi.org/10.2903/ j.efsa.2017.4787.

Abour H. Cherif, JoElla E. Siuda, Sana Kassem, Stefanos Gialamas, 2017. Which Sweetener Is Best for Yeast? An Inquiry-Based Learning for Conceptual Change. Journal of Education and Practice www.iiste.org. 
Vol.8, No.2.

Flushce G., Folchert S., Danielson T., Desandre N., Franks D. 2015. The use of rice as a sugar source in yeast fermentation: will yeast take the bait? Journal of Introductory Biology Investigations. 3. 2.

Graeme M Walker and graham G Stewart, 2016. Saccharomyces cerevisiae in the production of fermented beverages. Beverages, $\quad 2, \quad 30$ : 10.3390/beverages2040030.

Marissa Fennell, Casady Fuzzell, Kelli Haworth, Meelyn Pandit, 2016. Artificial Sweetener Has Higher Ethanol Production Compared to Organic Carbohydrates. Journal of Introductory Biology Investigations. Vol 4, No 3

M.F. Gliemmoa, A.M. Calvin, Tamasib, L.N. Gerschensona, C.A. Camposa, 2007. Interactions between aspartame, glucose and xylitol in aqueous systems containing potassium sorbate. Elsevier. LWT 41 (2008) 611-619.

Tilak W. Nagodawithana, Carmine castello and Keith H. Steinkaus, 1974. Effect of dissolved oxygen, temperature, initial cell count and sugar concentration on the viability of saccharomyces cerevisiae in rapid fermentations. Applied Microbiology, 28(3): 383-391.

R. C. Jones and J. S. Hough, 1970. The effect of temperature on the metabolism of
Baker's yeast growing on continuous culture. J. Gen. Microbiol, 60, 107116.

Rebecca J. Brown, Mary Ann De Banate and Kristina I. Rother, 2010. Artificial sweetener: A systematic review of metabolic effect in youth. Int $\mathbf{J}$ Pediatr Obes, 5(4): 305- 312.

Vikas purohit and Sundeep mishra, 2018. The truth about artificial sweeteners- Are the good for diabetics? Indian Heart Journal, Elsevier. vol. 70, issue 1, p 197-199.

Suzanne M. de la Monte and Ming Tong, 2015. Mechanism of Nitrosaminemediated Neurodegeneration: Potential relevance to sporadic Alzheimer's Disease. HHS Public Access. 17(4) : 817- 825.

Sanchari Chattopadhyay, Utpal Raychaudhuri and Runu Chakraborty, 2014. Artificial sweeteners - a review. J Food Sci Technol. 51(4): 611-621.

Cheng Wei Lui, Yu Sung, Bo Ching Chen and Hung Yu Lai, 2014. Effect of nitrogen fertilizers on the growth and nitrate content of lettuce (Lactuca sativa L.). Environment research and public health, 11(4): 4427-4440.

Polakowski T. M. 2008. The effect of different sugars on the rate of fermentation in yeast. Ohio Journal of Science. 108. 1. 30.

\section{How to cite this article:}

Krishna Chaitanya Naredla, Seema Gupta and Maisnam Jaya Devi. 2019. Investigating the Effects of Natural and Artificial Sugars on the Rate of Growth and Respiration in Saccharomyces cerevisiae. Int.J.Curr.Microbiol.App.Sci. 8(01): 1978-1983. doi: https://doi.org/10.20546/ijcmas.2019.801.207 